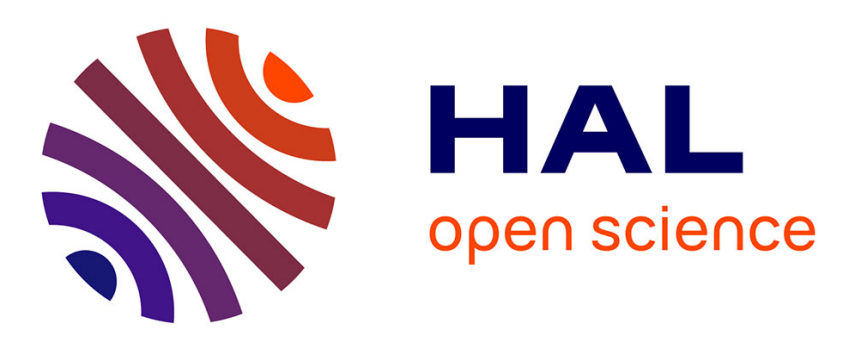

\title{
Addressing the stochasticity of nucleation: Practical approaches
}

N. Candoni, Z. Hammadi, R. Grossier, Manuel Ildefonso, R. Morin, S. Veesler

\section{To cite this version:}

N. Candoni, Z. Hammadi, R. Grossier, Manuel Ildefonso, R. Morin, et al.. Addressing the stochasticity of nucleation: Practical approaches. Rui Tamura, Mikiji Miyata. Advances in Organic Crystal Chemistry, Springer, pp.95-113, 2015, 978-4-431-55554-4. 10.1007/978-4-431-55555-1_6 . hal-01803259

\section{HAL Id: hal-01803259 \\ https://hal.science/hal-01803259}

Submitted on 30 May 2018

HAL is a multi-disciplinary open access archive for the deposit and dissemination of scientific research documents, whether they are published or not. The documents may come from teaching and research institutions in France or abroad, or from public or private research centers.
L'archive ouverte pluridisciplinaire $\mathbf{H A L}$, est destinée au dépôt et à la diffusion de documents scientifiques de niveau recherche, publiés ou non, émanant des établissements d'enseignement et de recherche français ou étrangers, des laboratoires publics ou privés. 


\title{
Addressing the stochasticity of nucleation: practical approaches
}

\author{
Nadine Candoni ${ }^{1}$, Zoubida Hammadi ${ }^{1}$, Romain Grossier ${ }^{1}$, Manuel Ildefonso ${ }^{2}$, Shuheng \\ Zhang $^{1}$, Roger Morin ${ }^{1}$, Stéphane Veesler ${ }^{1}$ \\ ${ }^{1}$ Aix-Marseille Université, CNRS, CINaM UMR 7325, 13288 Marseille, France \\ ${ }^{2}$ Université de Pau et des Pays de l'Adour, CNRS, LFC-R UMR 5150, 64012 Pau, France
}

\begin{abstract}
This chapter presents different practical ways to address nucleation stochasticity. The methods use either statistical studies on spontaneous nucleation or local control of nucleation. Techniques developed in our laboratory are described: droplet-based microfluidics, micro-injectors in oil, external electrical or mechanical fields in confined systems. Results of nucleation kinetics obtained on various molecules, are presented in terms of metastable zone, critical supersaturation, nucleation rate, induction time, interfacial energy of the critical nucleus. These practical approaches show considerable potential to increase understanding and control of the nucleation mechanism.
\end{abstract}

Keywords : spontaneous nucleation, induced nucleation, microfluidics, confinement, external fields.

\section{Introduction}

In crystallization of materials such as pharmaceuticals, proteins, or nanomaterials, the most important properties are crystal morphology, habit, size distribution and phases. The control of these properties demands an understanding of the mechanisms governing the successive steps of nucleation and growth. Although a great deal is known about crystal growth, considerably less is known about crystal nucleation because of the difficulty of directly observing the nuclei. Indeed, the first nuclei are defined by Kashiev as randomly generated nanoscopic new phases "that have the ability for irreversible overgrowth to macroscopic size" [1]. Hence nucleation is a stochastic phenomenon and the probability of observing the first nuclei is very low, for spatial and temporal reasons.

In this chapter we present practical ways of addressing the stochasticity of nucleation [2]. The first idea consists in performing many spontaneous nucleation experiments, and hoping that the law of large numbers permits us to get a sharper picture of nucleation. Observation is enhanced by reducing the volume of crystallization [3], and hence the number of crystals. The techniques presented here are droplet-based microfluidics [4-9] and micro-injection in oil [1013]. The second idea consists in inducing nucleation where and when we choose controlling nucleation, which is no longer spontaneous. In this case, the techniques combine confinement of nucleation with localized external fields, such as electrical [14-15] and mechanical fields [16].

The purpose of this chapter is to show what these techniques currently offer in terms of nucleation kinetics measurements and detection of the critical nucleus. The main questions are: what are the factors that influence nucleation and how they are revealed by these techniques? Since we explore nucleation from a purely practical point of view, we do not consider any a priori assumption on the nucleation mechanism. We will present practical examples with mineral, organic anud biological molecules confirming that nucleation mechanisms are identical for all molecules [17]. 


\section{Theoretical background}

The following is a brief reminder of nucleation theory and experimental parameters, introducing all the properties accessible with our techniques.

\subsection{Critical nucleus}

Until recently, solution nucleation has been described solely by the classical nucleation theory (CNT), a theory derived from nucleation of droplets in the bulk of pure supersaturated vapors [18]. It considers that once a cluster has reached a critical size, nucleation starts. Hence the first nucleus is called the critical nucleus. In this model, the critical nucleus appears by densification of molecules and nucleation of a solid phase at the same time. Therefore the transition from the liquid phase to the solid phase is described by one order parameter, i.e. density. According to CNT, the critical size $r^{*}$ is given by the Gibbs-Thomson equation:

$$
r^{*}=\frac{2 \Omega \gamma}{k T \ln \beta} \quad \text { equation (1) }
$$

With $\Omega\left(\mathrm{m}^{3}\right)$ the volume of a molecule inside the crystal, $\gamma\left(\mathrm{J} \cdot \mathrm{m}^{-2}\right)$ the interfacial energy of the critical nucleus, $\mathrm{k}$ the Boltzmann constant $\left(\mathrm{J} . \mathrm{K}^{-1}\right), \mathrm{T}$ the temperature $(\mathrm{K})$ and $\beta$ the supersaturation $\left(\mathrm{C} / \mathrm{C}_{\mathrm{s}}\right) . \mathrm{C}$ is the concentration of the solute in solution and $\mathrm{C}_{\mathrm{s}}$ the equilibrium concentration. Hence, if $\beta>1$, the crystal grows, if $\beta<1$ the crystal dissolves, and if $\beta=1$ crystals and solution are at equilibrium.

While this theory has the advantage of simplicity, some discrepancies (in predicting nucleation frequencies) have been observed with experiments [3, 19-22]. Consequently, a more complicated two-step mechanism was proposed: first, formation of a dense phase of clusters on the model of a liquid-liquid phase separation and second, organization of these clusters into structured clusters [23-25]. This theory is based on two order parameters, density and structure [26]. Subsequently, a third nucleation theory was proposed linked to biomineralization, consisting of a nucleation via stable prenucleation clusters [27]. For reviews see 28, 29. Concerning experimental discrepancies, different explanations are proposed. For instance, Knezic et al. [30] suggested that macromolecules take longer to rearrange themselves into crystalline nuclei than smaller molecules (this rearrangement step is not directly taken into account in CNT). Hence, the limiting step, densification or structuration, will depend on the solute.

It must be pointed out that experimental observations and interpretations are influenced by the fact that the location, the nature and the lifetime of the dense phase and/or the critical nucleus are currently unknown.

\subsection{Nucleation kinetics}

In this chapter we are looking at primary nucleation, i.e. the appearance of crystals in a solution which contains no crystals of that phase. The nucleation kinetics is commonly described by the nucleation rate $J$, which is the number of nuclei that form in the supersaturated solution per unit of volume and per unit of time [1, 31-33]. Theoretically, the nucleation rate $J$ is determined by CNT derived from the Gibbs treatment of droplet nucleation [18]. The general equation for primary nucleation is expressed as equation (2) [34]:

$$
I=K_{0} \exp \left(\frac{\Delta G^{*}}{k T}\right) \text { equation (2) }
$$

with $\mathrm{K}_{0}$ the kinetic factor $\left(\mathrm{m}^{-3} \cdot \mathrm{s}^{-1}\right)$ and $\Delta \mathrm{G}^{*}$ the activation free energy $(\mathrm{J})$. 
CNT assumes a spherical form for the critical nucleus as mentioned by Fletcher [35]. In the general case of heterogeneous or homogeneous nucleation, a constant shape for the cluster (here spherical) is assumed, which depends on the possible presence of foreign substances: foreign molecules or particles, bubbles, crystallizer walls, liquid-liquid or liquid-air interfaces. Hence the value of $\Delta G^{*}$ depends on surface interactions, which are taken into account by introducing an effective interfacial energy $\gamma_{\mathrm{ef}}$ of the critical nucleus and $\mathrm{J}$ is given by equation (3) [4]:

$$
J=K_{0} \exp \left(-\frac{16 \pi}{3} \frac{\Omega^{2} \gamma_{\mathrm{ef}}^{3}}{(k T)^{3} \ln ^{2} \beta}\right)
$$

equation (3)

\subsection{Critical supersaturation}

According to equation (3), there is a zone in the supersaturated region $(\beta>1)$ where no nucleation occurs for a given time in a given volume. This is the metastable zone [36], which is delimited by the critical supersaturation (CS) for a given temperature or the temperature of metastable zone (TMZ) for a given concentration (Fig. 1).

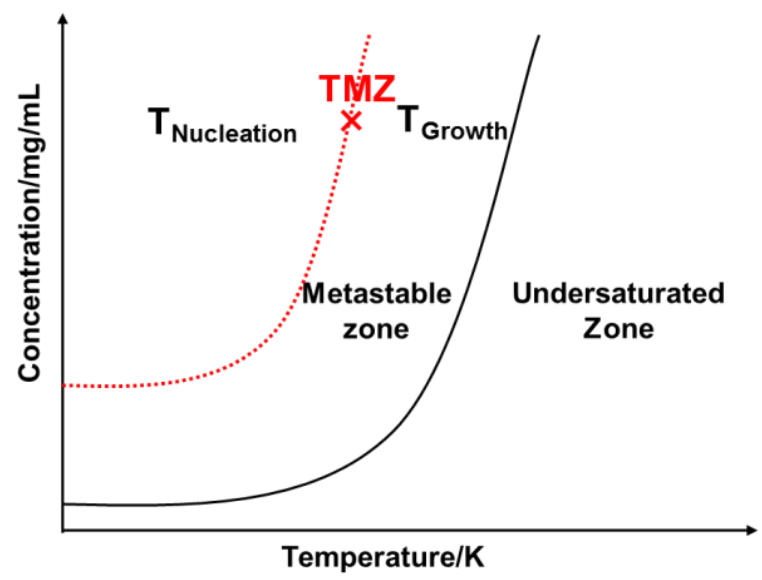

Fig. 1 Phase diagram of supersaturation vs temperature, with the solubility curve (-) and the metastable zone $(\cdots)$.

In the nucleation zone or zone of spontaneous nucleation, nucleation (i.e. the appearance of crystals) is promoted; in the metastable zone, growth (i.e. the subsequent expansion of the crystal) is promoted.

\subsection{Experimental considerations}

In practice, the two usual methods used to determine the nucleation rate $\mathrm{J}$ are:

- Measurement of the induction time, i.e. the time it takes to form a detectable amount of the new crystalline phase after a given supersaturation $\beta$ is established. However nuclei must grow to a minimum size before they are observable; thus the induction time includes the nucleation time and the time required for growth to a detectable size. The supersaturation range over which the experiment can be performed is rather limited due to the metastable zone. When $\beta$ is too small heterogeneous nucleation may replace homogeneous nucleation. When $\beta$ is too large, induction times are difficult to measure because they are too short. In practice, we are able to measure the induction time in the vicinity of the metastable zone limit 
and we could reasonably assume that the growing period can be considered negligible compared to the nucleation period.

- The Double Pulse Technique (DPT), which permits nucleation and growth to be separated [37-39]. At the beginning of a run, the solution is loaded at a temperature chosen to prevent nucleation of crystals. Then the temperature is lowered (respectively raised if the solubility is reverse with $\mathrm{T}$ ) to a selected $\mathrm{T}_{1}$ at which nucleation occurs. After a period of $\Delta \mathrm{t}_{1}$ (nucleation time), the temperature is raised (respectively lowered if the solubility is reverse with $T$ ) from the nucleation temperature $T_{1}$ to the growth temperature $T_{2}$ in the metastable zone. At $\mathrm{T}_{2}$, crystals already formed can grow to detectable dimensions. The nucleation rate, at a given concentration $C$ and temperature, is given by the variation of the amount of crystals nucleated as a function of nucleation time.

\section{Studying nucleation, an unpredictable phenomenon}

As we have seen above, nucleation plays a fundamental role in the physical properties of crystals. However the unpredictability of the spatial and temporal location of the critical nucleus makes its study difficult. In this part we address the problem of the stochasticity of nucleation in two ways: first by carrying out statistical studies on single crystals spontaneously nucleated; second by inducing nucleation.

\subsection{Statistical studies of spontaneous nucleation}

For statistical studies, a large number of samples in the same condition are required. In large volumes, it is difficult to accurately detect crystals as they are formed, so sample volumes need to be limited. Therefore the idea is to reduce the volume of nucleation to the $\mathrm{nL}-\mathrm{pL}$ range. Hence the number of crystals nucleating and the zone of observation are reduced, thus increasing the resolution of the detection. Moreover, this has the advantage of decreasing the quantity of molecules while speeding up heat and mass transfer [40]. Volume reduction to $\mathrm{nL}$ is realized using droplet-based microfluidics. Then $\mathrm{pL}-\mathrm{fL}$ volumes are obtained by micro-injection methods.

\subsubsection{Nucleation in nanoliter droplets}

Droplet-based microfluidics permits series of nanolilter droplets to be generated by injecting the crystallization solution in a non-miscible continuous phase and without using surfactant. Droplets are mono-dispersed (in size, composition, frequency) nano-crystallizers isolated from each other and in which crystallization can occur independently. Hundreds of droplets can be generated per condition, leading to a large number of samples for statistical studies, while consuming only small quantities of material.

- Microfluidic devices: (i) molded in PDMS and adapted from Salmon [41-43] and (ii) composed of Teflon tubing with junctions in PEEK (Fig. 2a-b). The continuous phase is silicone or fluorinated oil according to the solvent of crystallization (discontinuous phase). The crystallization solvent is water in PDMS systems. This microfluidic system thus becomes compatible with almost all solvents, since Teflon tubing is used instead of PDMS chips (Fig. 2c). Crystals of various organic molecules obtained in aqueous and organic solvents are presented in figs $2 \mathrm{~d}-2 \mathrm{~h} \mathrm{[6].}$ 


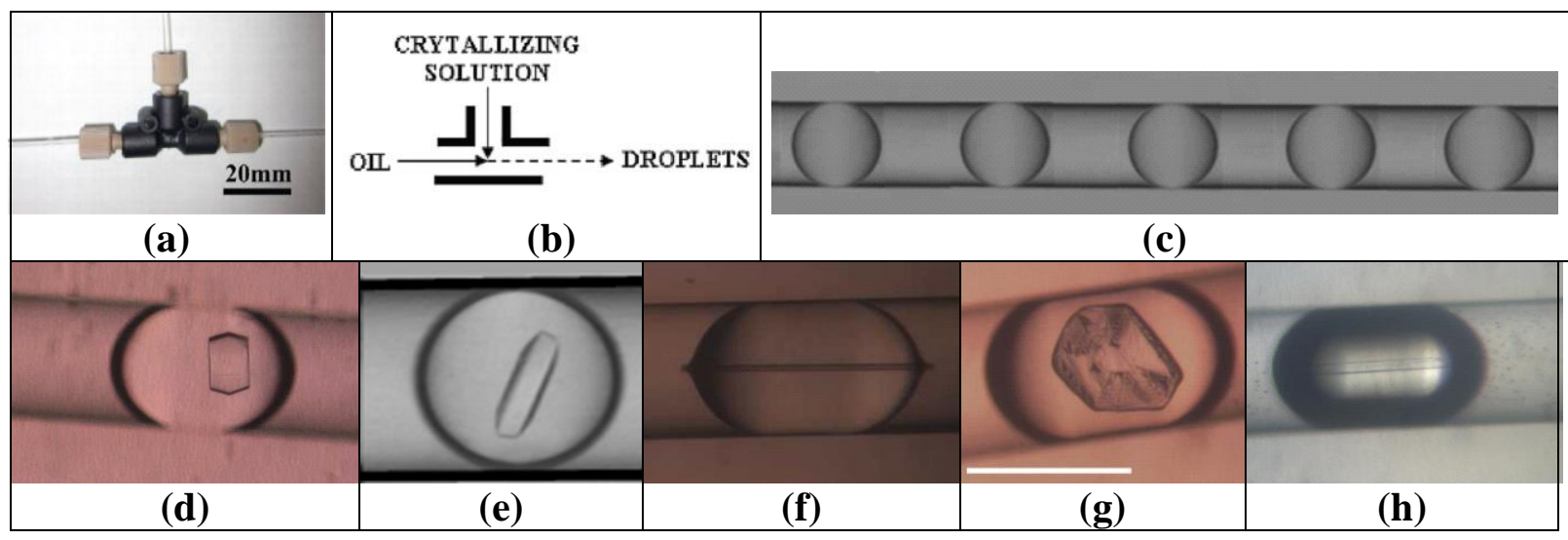

Fig. 2 (a) Photo and (b) scheme of a T-junction made of PEEK; (c) Photo of isolated droplets of $60 \mathrm{~nL}$ volume of crystallization solution separated by fluorinated oil in Teflon tubing of $500 \mu \mathrm{m}$ diameter, (d) Lysozyme crystals in aqueous droplets, (e) Rasburicase crystal in a viscous medium, (f) Caffeine crystals in ethanol droplets, (g) Isonicotinamide crystal in an ethanol droplet and (h) Isonicotinamide crystals in nitrobenzene droplets in Teflon tubing of 500 $\mu \mathrm{m}$ diameter [6]. Copyright 2012 American Chemical Society.

The microfluidic set-up [7] in Fig. 3 allows us to determine the properties of nucleation described above theoretically: metastable zone width, nucleation rate $\mathrm{J}$, effective interfacial energy $\gamma_{\mathrm{ef}}$ of the critical nucleus and polymorphism.

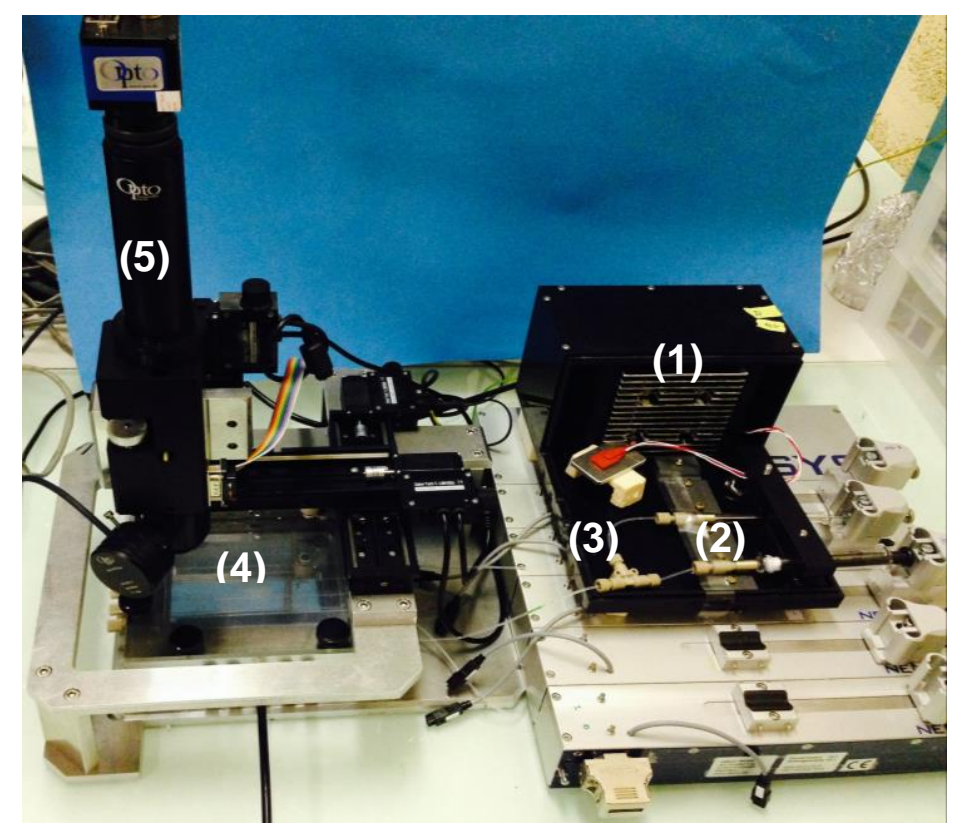

Fig. 3 The microfluidique set-up: (1) and (2) Thermostatted Syringe-pumps, (3) T-junction, (4) Thermostatted sample-holder, (5) Camera + Zoom on XYZ stage. 
- Metastable zone width: evaluated from measurements of the temperature of the metastable zone (TMZ) illustrated in Fig. 1. This is the maximum temperature above which the supersaturated solution does not lose its metastability over a given time [36]. For a given concentration, TMZ is determined by storing solutions at given temperatures for a given time (here 20h). During this time if $T<\mathrm{TMZ}$, crystals nucleate and if $T>\mathrm{TMZ}$, no crystal appears. Hence TMZ at which there is no crystal is extrapolated (interpolation) from a plot of the average number of crystals versus temperature. Moreover a rapid screening of the solubility diagram (black curve of Fig. 4) can be carried out as shown by Laval et al [41-42].

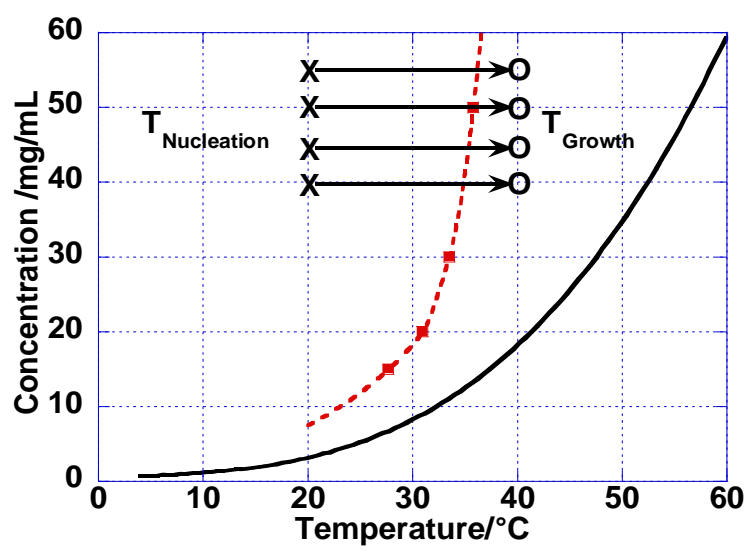

Fig. 4 Metastable zone [5] (- - -) and solubility curve [44] (----) of tetragonal lysozyme (0.7M $\mathrm{NaCl}, \mathrm{pH}=4.5)$, lines improve legibility. Points $(\mathrm{x})$ and $(\mathrm{O})$ indicate nucleation and growth conditions, respectively. Copyright 2011 American Chemical Society.

- Nucleation rate: determined by using the Double Pulse Technique (DPT), based on the phase diagram of figure 4 . Crystals are nucleated at a $\mathrm{T}_{\text {Nucleation }}$ outside the metastable zone (below TMZ) and for a given nucleation time $\Delta t$; then, the crystal are stored at $T_{\text {Growth }}$ in the metastable zone, where the crystals already formed grow to detectable dimensions and are counted. From the variation of the average number of crystals nucleated as a function of $\Delta t$, the steady-state primary nucleation rate $\mathbf{J}$ is determined for given temperature and concentration. Our results obtained in nucleation volumes of $250 \mathrm{~nL}$ of lysozyme are summarized in Fig. 5.

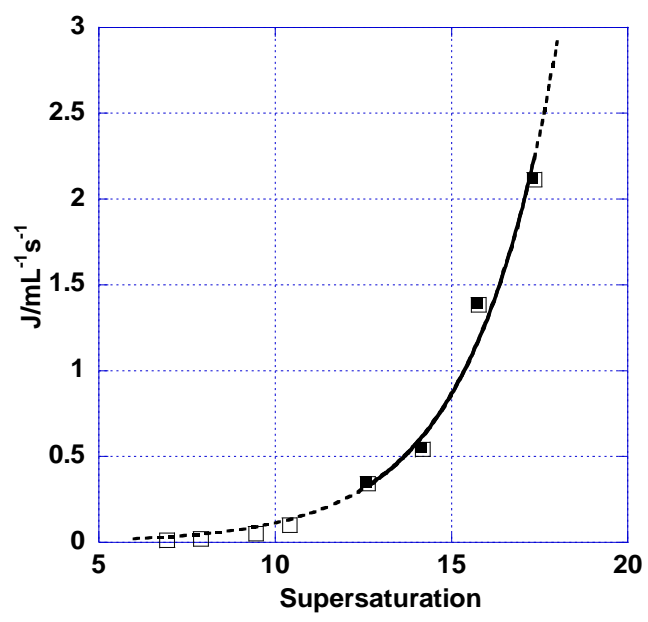

Fig. 5 Lysozyme nucleation rate vs supersaturation, (ם) data from our study at $20^{\circ} \mathrm{C}$ [5] and ( $\square$ ) data from Galkin and Vekilov, at $12.6^{\circ} \mathrm{C}$ [21-22]. Copyright 2011 American Chemical Society. 
As shown in Fig. 5, our kinetic data are in good agreement with the data of Galkin and Vekilov [21-22] obtained for the same $\mathrm{NaCl}$ concentration at $12.6^{\circ} \mathrm{C}$, using droplets of $1 \mu \mathrm{L}$ suspended in Teflon wells. Moreover, the supersaturation range experimentally accessible when reducing the volume of nucleation to the $\mathrm{nL}-\mathrm{pL}$ range is increased for kinetic [5] and thermodynamic [14] reasons (see part 3.2.).

- Effective interfacial energy $\gamma_{\text {ef }}$ computed from eq. 3 shows that primary nucleation is not influenced by the container material (Fig. 5). Indeed, the oil creates a "containerless" environment, as pointed out by Chayen [45]. However, $\gamma_{\text {ef }}$ determined in fluorinated oil and in silicone oil (Tab. 1) demonstrate the influence of the droplet-oil interface in droplet-based microfluidics [4].

\begin{tabular}{|c|c|c|c|c|}
\hline & Ildefonso et al. $^{5}$ & Vekilov group $^{21-22}$ & Ildefonso et al. $^{4}$ & Fraden group $^{46}$ \\
\hline device & PDMS & Teflon wells & Teflon & PDMS \\
\hline oil & silicone & silicone & fluorinated & fluorinated \\
\hline$\gamma_{\mathrm{ef}}\left(\mathrm{mJ} / \mathrm{m}^{2}\right)$ & $0.62(0.13)$ & 0.56 & $0.88(0.05)$ & 0.91 \\
\hline
\end{tabular}

Tab. 1 Effective interfacial energy $\gamma_{\mathrm{ef}}$ of the critical nucleus determined for oils of different chemical natures, by different authors. The deviation in absolute is given in parentheses; errors are not available in 21, 22 and 46.

- Polymorphism: droplet-based microfluidics is adapted for detection of new phases for statistical reasons. For instance, different polymorphs can nucleate, the most stable polymorph corresponding to the minimal free energy of the system. Moreover, according to Ostwald's rule of states [47], a chemical system does not tend directly towards the most stable state, but rather tends towards the closest metastable state. Hence performing many spontaneous nucleation experiments increase the probability of observing an event. In the case of lysozyme, we observed a metastable phase, the sea urchin-like phase, in 6 droplets out of 237 (Fig. 6) [8]. It was already known [48-49], but is not easy to observe in mL crystallizers. Moreover, because of mononuclear nucleation, the nucleated crystal of the metastable phase cannot easily follow a solution-mediated phase transition to a more stable phase. Thus small volumes of droplets seem to "freeze" the metastable phase [50]. Lastly, the higher supersaturations reached in microfluidics due to volume reduction increase the probability of nucleating a metastable phase [51].

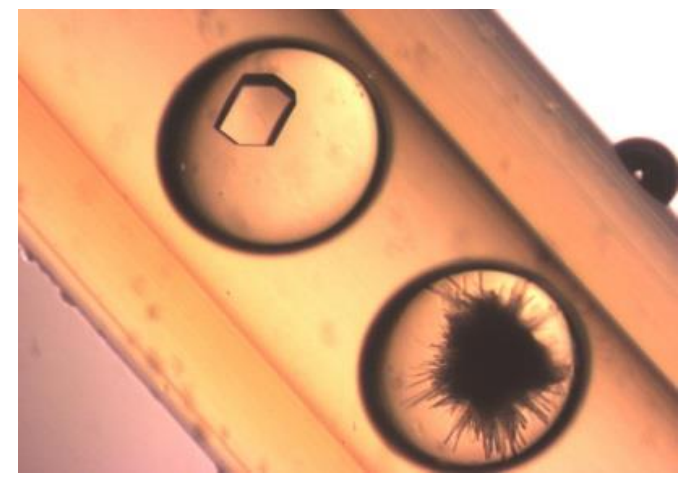

Fig. 6 Droplets of lysozyme solutions (20 mg/mL, $0.7 \mathrm{M} \mathrm{NaCl}-\mathrm{pH} 4.5)$ in Teflon tubing of $500 \mu \mathrm{m}$ diameter, observed at $20^{\circ} \mathrm{C}$ after storage for $20 \mathrm{~h}$ at $6^{\circ} \mathrm{C}$ : the tetragonal form top left and the sea urchin-like form bottom right [8]. 
In screening the crystallization conditions of rasburicase, the same experimental conditions produce different crystal habits and phases. Moreover, the two polymorphs known for rasburicase [52] appear simultaneously in droplets (Fig. 7) [9]. This concomitant nucleation occurs mainly because the difference between their solubilities is weak.

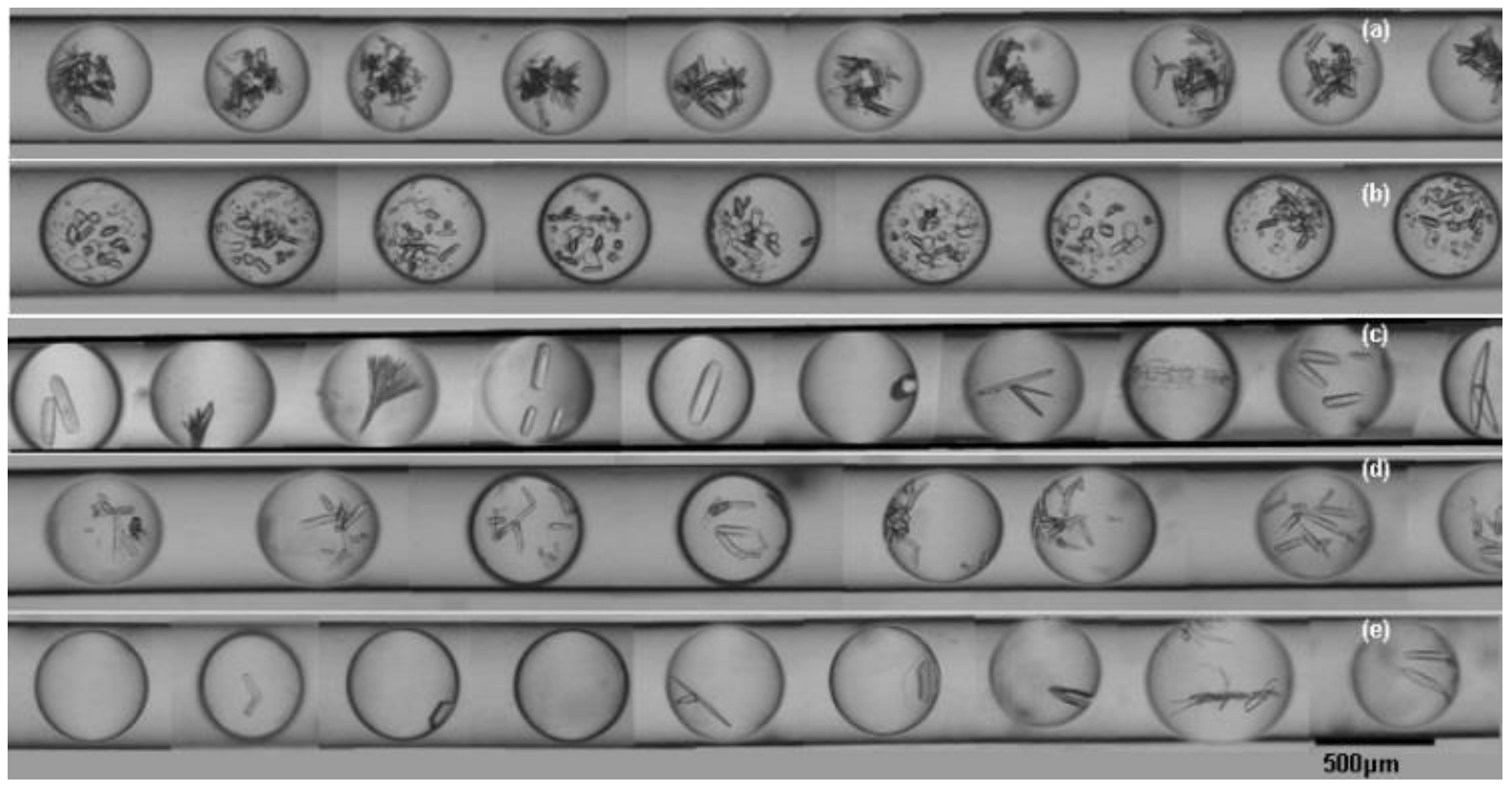

Fig. 7 Photos of crystals of rasburicase obtained in $65 \mathrm{~nL}$ droplets: each line corresponds to the same crystallization conditions (a) $10 \% \mathrm{PEG}, 10 \mu \mathrm{g} / \mu \mathrm{L}$ rasburicase, at $5^{\circ} \mathrm{C}$. (b) $10 \% \mathrm{PEG}$, $10 \mu \mathrm{g} / \mu \mathrm{L}$ rasburicase, at $20^{\circ} \mathrm{C}$. (c) $5 \% \mathrm{PEG}, 10 \mu \mathrm{g} / \mu \mathrm{l}$ rasburicase, at $5^{\circ} \mathrm{C}$. (d) $7.5 \% \mathrm{PEG}$, $5 \mu \mathrm{g} / \mu \mathrm{L}$ rasburicase, at $5^{\circ} \mathrm{C}$. (e) $7.5 \%$ de $P E G, 5 \mu \mathrm{g} / \mu \mathrm{L}$ rasburicase, at $20^{\circ} \mathrm{C}$ [9]. Copyright 2015 American Chemical Society (paper submitted).

Fig. 6 and 7 highlight the importance of performing statistical studies through a large number of experiments per condition because of the stochasticity of nucleation. Statistically each crystallization condition should be tested from 50 to 100 times in order to decide whether the result (obtaining crystals, targeted polymorph(s)) is positive or negative. This is the main problem with the conventional crystallization robots, where typically a few hundred different conditions are tested only 1 to 5 times each, leading often to non-reproducible results.

To summarize, droplet-based microfluidics is an accurate method for studying nucleation kinetics due to statistical approach, exploring one experimental condition in hundreds of identical droplets. Moreover, as droplet volumes are nanoliter, this statistical approach requires only a few milligrams of molecules.

\subsubsection{Nucleation in picoliter to femtoliter droplets}

Small-droplet generation with micro-pipets or micro-injectors renders micro-droplets accessible, and makes the size range easier to control compared to microfluidics, which is limited by channel size (hardware limitation). However, statistical analysis cannot be carried out with single micro-droplet injection [40, 53-54]. 
- The fluidic device developed in our laboratory [10] (Fig. 8a) generates arrayed aqueous phase micro-droplets through a layer of inert oil (sessile geometry), without using surfactant. Up to thousands of micro-droplets can be generated, with volumes ranging from nanoliter to femtoliter (Fig. 8b), thus allowing statistical studies of nucleation.

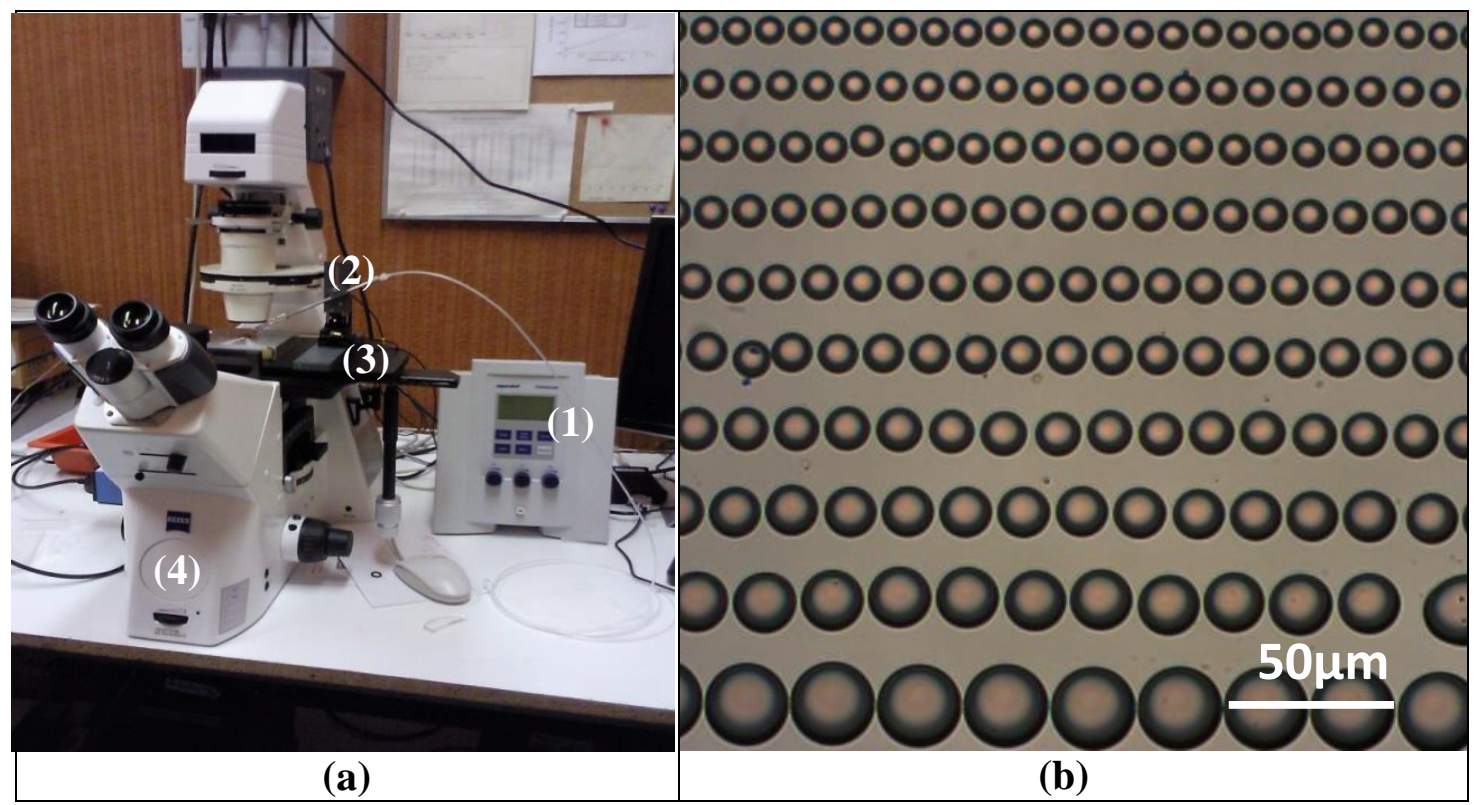

Fig. 8 (a) The micro-injector in oil set-up: (1) injector, (2) glass micropipette, (3) XYZ miniature translation stages for injector and (4) microscope; (b) Array of monodisperse water droplets with volumes ranging from nanoliter to femtoliter [10]. (a) Copyright 2010 Elsevier and (b) with permission from [10]. Copyright 2011 AIP Publishing LLC

- The droplet-contraction method is used to generate supersaturation and thus to create crystals, as we can see with picoliter droplets of $\mathrm{NaCl}$ solution in Fig. 9a [16]. Indeed, at this scale, droplets slowly evaporate, linearly decreasing the volume (Fig. 9b) [11]. Hence the concentration, which is given by the refractive index [12], increases until it exceeds the critical supersaturation, i.e. the supersaturation required to nucleate a new phase [55]. Then single crystals nucleate (Fig. 9c).

Fig. 9 (a) Array of droplets $(\sim 300 \mathrm{pL})$ of $\mathrm{NaCl}$ solutions at $\beta=0.1$ generated through a layer of inert DMS oil. (b) Contraction of droplets due to evaporation of aqueous phase in oil. (c) Nucleation of single crystals in every droplet [16]. 
- The induction time can be measured with the droplet-contraction method because the growth rate is so fast that the time required for the newly-formed nuclei to grow to a detectable size is negligible with regard to the induction time [13]. Thus, the time when a detectable crystal is observed can be considered as the induction time. Moreover, the smaller the volume, the faster the evaporation rate and the faster the nucleation (Fig. 10) [16].

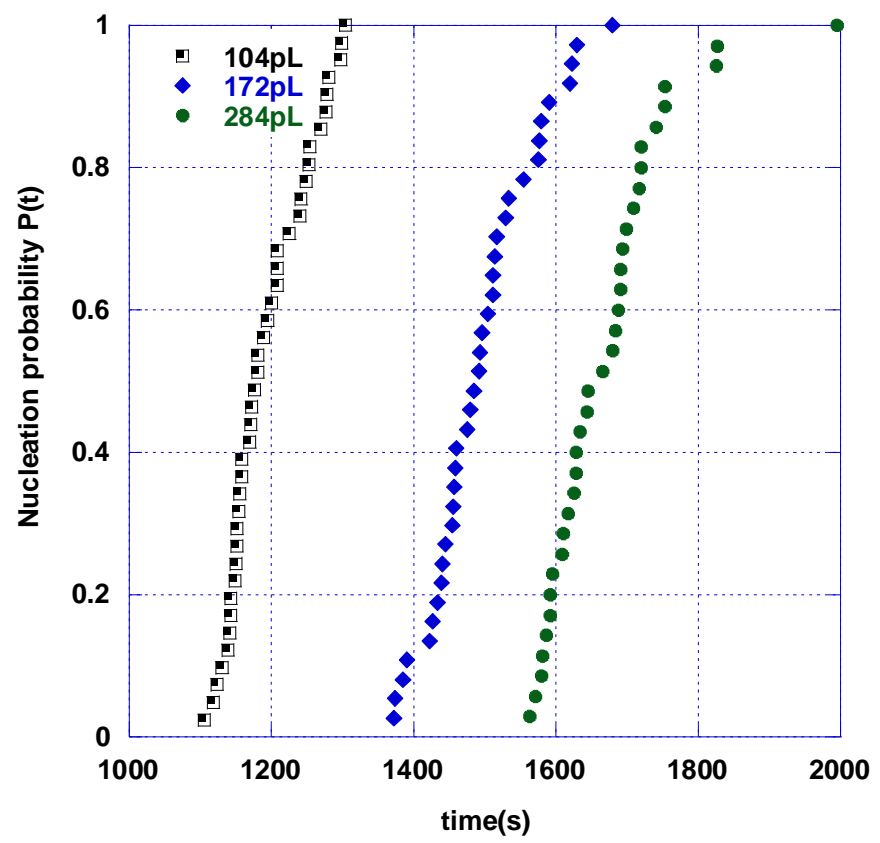

Fig. 10 Nucleation probability $\mathrm{P}(\mathrm{t})$ vs time. $\mathrm{P}(\mathrm{t})$ represent the normalized fraction of nucleated microdroplets for droplet diameters of $(\square) 104 \mathrm{pL},(\diamond) 172 \mathrm{pL}$ and (๑) $284 \mathrm{pL}$ [16]. Reproduced from Ref [16] with permission from The Royal Society of Chemistry

To summarize, this technique of droplet micro-injection in oil combines the advantage of channeled microfluidic techniques, generating thousands of droplets, with the advantage of micropipette techniques: control over size, micro-droplet accessibility and contraction of droplets in oil to increase supersaturation. Both of these techniques involve easy-to-build devices and are easy to use. Moreover, it must be noted that they require no surfactant that might affect crystallization.

\subsection{Influence of volume on nucleation}

Reducing the volume of crystallization raises several questions: How do kinetic parameters such as the metastable zone and the critical supersaturation vary? Does CNT, which supposes an infinite reservoir of molecules, i.e. a constant supersaturation during the nucleation event, remain valid in small volumes? What are the limitations of small volumes and how small?

- A kinetic limitation in small volumes arises from nucleation rate $\mathrm{J}$ (number of nucleus per unit of volume and per unit of time). $J$ is an intensive property (does not vary with volume). However $\mathbf{J}$ is inversely proportional to volume and proportional to induction time. Hence the smaller the volume, the longer the induction time. For instance, an induction time of $1 \mathrm{~s}$ in a volume of $1 \mathrm{~mL}$ becomes 11 days in a volume of $1 \mathrm{~nL}$. 
- A thermodynamic limitation appears because the critical size and energy barrier required for nucleation increase when the crystallization volume decreases [56]. When the volume decreases, the concentration in solution can no longer be considered constant during the nucleation process but is decreasing. In practice, this means that the reservoir of molecules is finite, in contradiction with CNT. Hence the critical supersaturation, where no nucleation can occur, increases with confinement (Fig. 11), widening the metastable zone [13, 56]. Thus "scale-down" requires creation of sufficient supersaturation within each droplet to ensure nucleation, with a risk of unwanted nucleation before droplet formation. In practice there is a "thermodynamic" influence of volume on nucleation from picoliter range down.

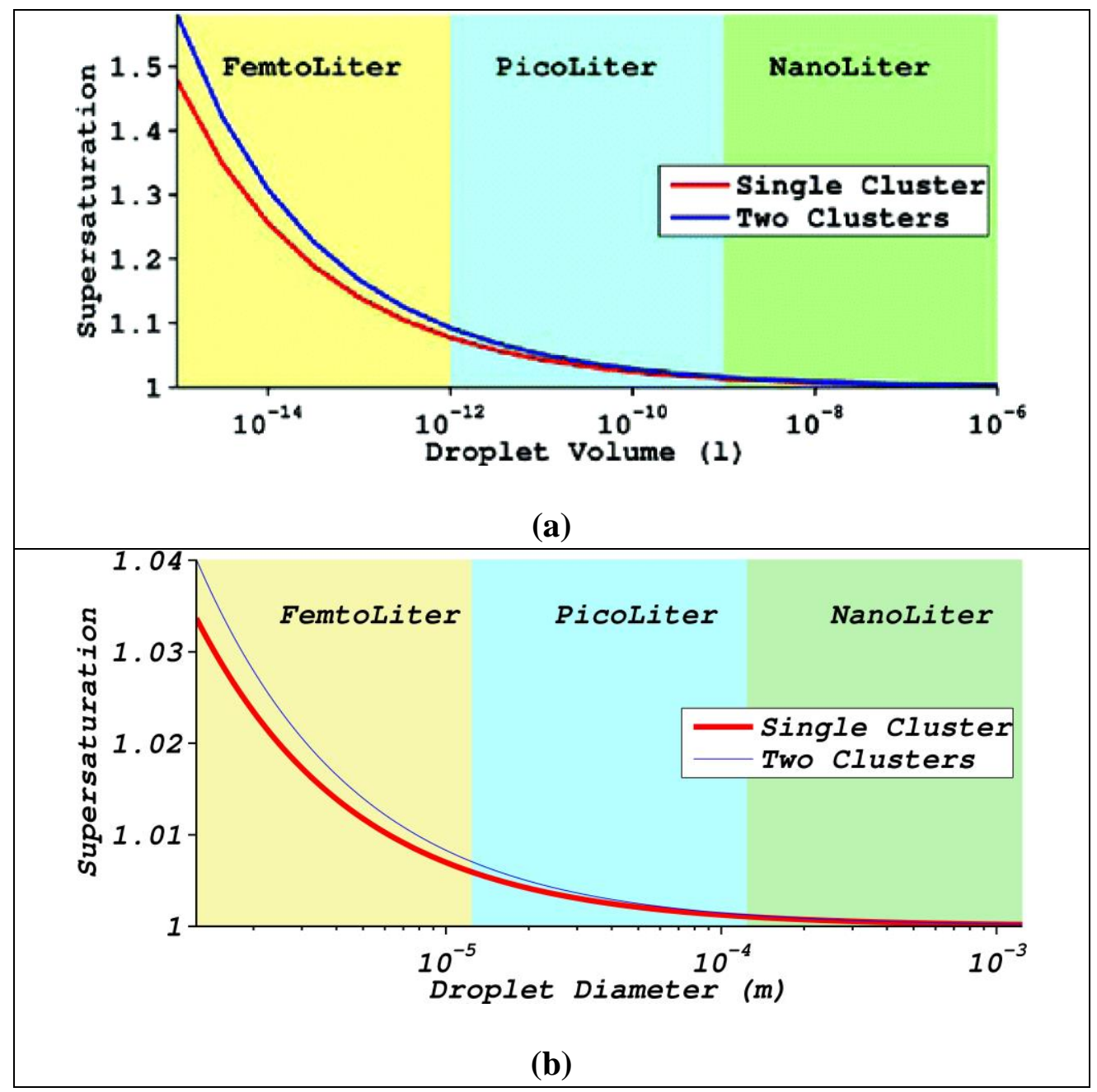

Fig. 11 Critical supersaturation (below which no nucleation can occur) vs droplet size, the red curve for a single nucleation event and the blue curve for a second event: (a) for lysozyme in $\mathrm{NaCl}$ solution [56]; (b) for $\mathrm{NaCl}$ [13]. (a) Copyright 2009 American Chemical Society and (b) Copyright 2010 Elsevier

To summarize, two effects occur depending on droplet volume: 1) for $\mathrm{nL}$ range droplets, a kinetic effect increases the induction time; 2) for pL-fL range droplets, the same kinetic effect is accompanied by a thermodynamic effect. In both cases these effects widen the metastable zone, hence requiring higher supersaturation for nucleation. However these effects stabilize high-supersaturated solutions, permitting critical clusters and nucleation to be controlled. 


\subsection{Predictive study of induced nucleation}

Nucleation can even be induced in the metastable zone, thus becoming predictive. In other words, we determine where and when it will occur. Nucleation is induced using an external field, the implications of which were highlighted by Voss [57], Oxtoby [58] and Revalor [59]. Two effects on the supersaturated solution are expected: molecular orientation and density fluctuation. As a general rule, any disturbance triggers nucleation once a highlysupersaturated metastable state has been achieved [60]. First, localization in time is achieved by triggering nucleation in a metastable solution with a localized mechanical contact or a localized electrical field. Then localization in space is achieved by either confining the nucleation volume or by controlling fluxes in non-convective gel medium.

\subsubsection{Mechanical action and confinement by volume}

- Coupling a mechanical action using a sharp tip with volume confinement by microinjector triggers nucleation, as previously shown. A structural transformation is thereby induced in a dense supersaturated droplet at precisely determined points and times (Fig. 12). Then the crystal grows at a rate greater than $200 \mu \mathrm{m} / \mathrm{s}$ for the first $20 \mathrm{~ms}$ [16].
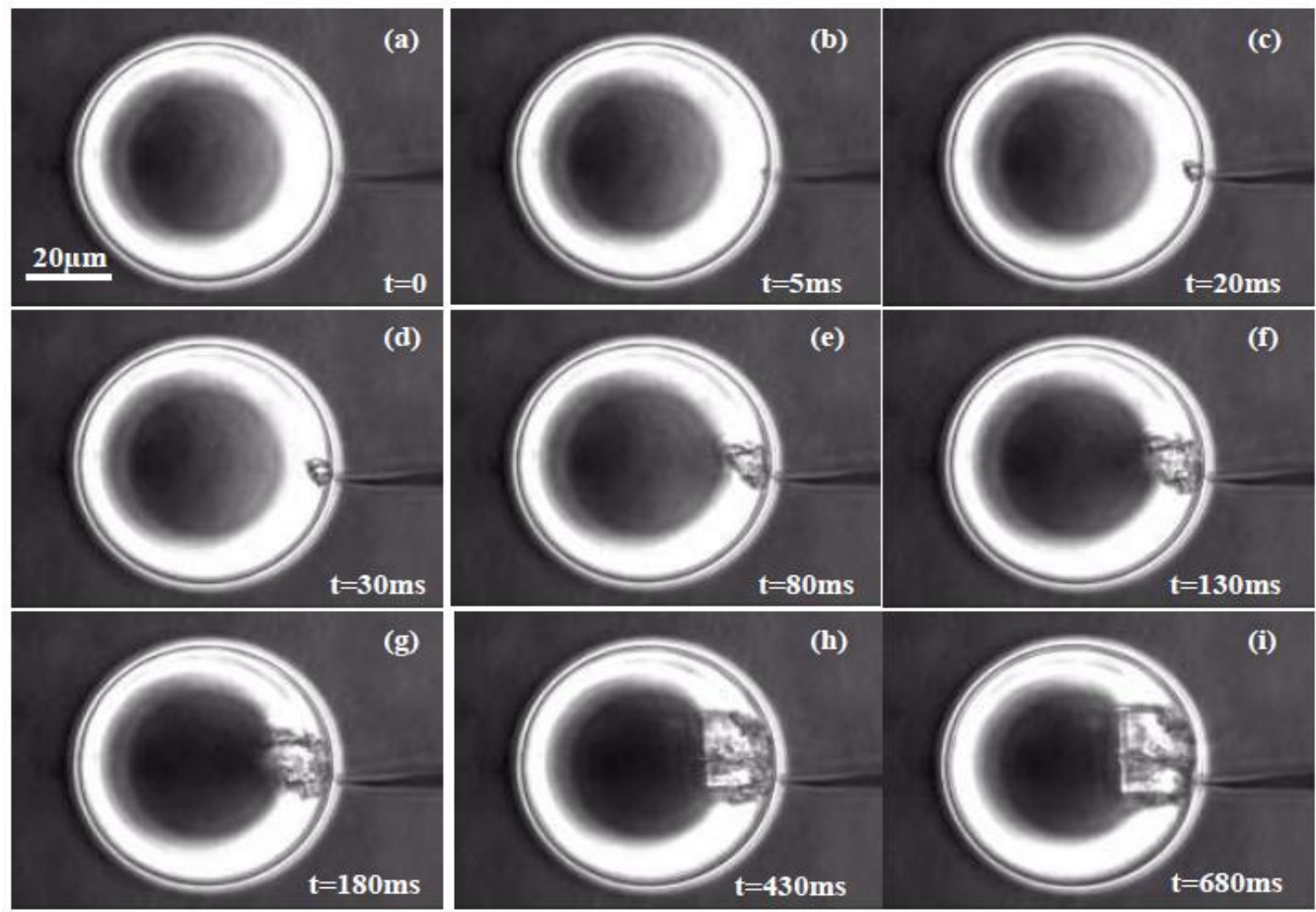

Fig. 12 Time sequence showing nucleation induced by a sharp tip touching a $95 \mathrm{pL}$ droplet of $\mathrm{NaCl}(\beta>1.24)$ and growth of $\mathrm{NaCl}$ in paraffin oil: (a) to (b) nucleation in less than $5 \mathrm{~ms}$, (c) to (i) growth [16]. Reproduced from Ref [16] with permission from The Royal Society of Chemistry

- The induction time is shorter than $5 \mathrm{~ms}$, corresponding to the time between tip contact and observation of nucleation (Fig. 12a-b). However the droplet is repeatedly tapped with the tip during the generation of supersaturation by water diffusion in order to launch nucleation. The crystal nucleated is rough and transition to a facetted crystal is observed in less than $1 \mathrm{~s}$, i.e. the transition between the nucleation form and the equilibrium form. In practice, the tip position being controlled with micromanipulators, the position of the critical nucleus can be determined with an accuracy of $16 \mathrm{~nm}$. 
- This method is suitable for a binary crystallization medium, with a solute and a solvent. The solute concentrates during droplet contraction and reaches high supersaturation. Then the solute nucleates due to the mechanical action. When the crystallization medium is ternary containing a solvent, a solute and a crystallization agent, e.g. protein crystallization, both solute and crystallization agent concentrate during droplet contraction and reach high supersaturation. Hence for kinetic reasons [61], the crystallization agent often nucleates first, before solute nucleation. Therefore, supersaturation must be achieved via a method other than droplet contraction.

\subsubsection{Electrical field and confinement by gel}

- Coupling a localized electric field generated by a sharp-tip electrode with a gel eliminating convection in a supersaturated metastable solution increases supersaturation at the tip apex and induces nucleation. A large electrical field [62] and large field gradients are created near the tip at low DC voltage, due to its nanometer size (Fig. 13).

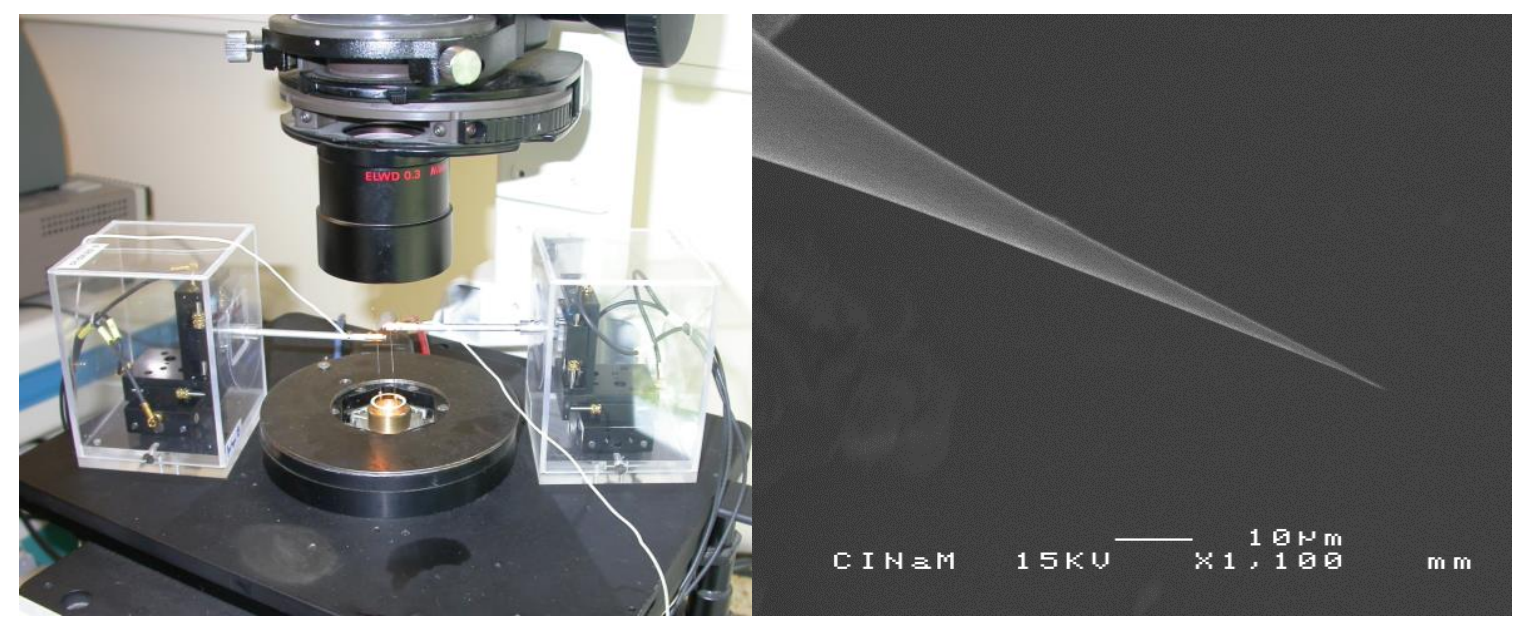

(a)

(b)

Fig. 13 (a) Temperature-controlled crystallization cell with 2 micromanipulators and (b) SEM image of a W-tip [2]. Reproduced from Ref [16] with permission from The Royal Society of Chemistry

The electrical field induces high current density inside the solution close to the region of high curvature [14]. The resulting flux accumulates molecules at a precise point in the vicinity of the tip apex [15] and the concentration gradient in solution tends to be counteracted by convection. However, the solution being in a gel, convection is eliminated and the confinement is kept in the vicinity of the tip until the critical supersaturation is reached. Then the crystal nucleates near the electrode tip [16].

- Induction time is measured by optical observation and by the evolution of the current in the solution. An example of nucleation induction in a supersaturated metastable solution of lysozyme in agarose gel is shown in Fig. 14. 


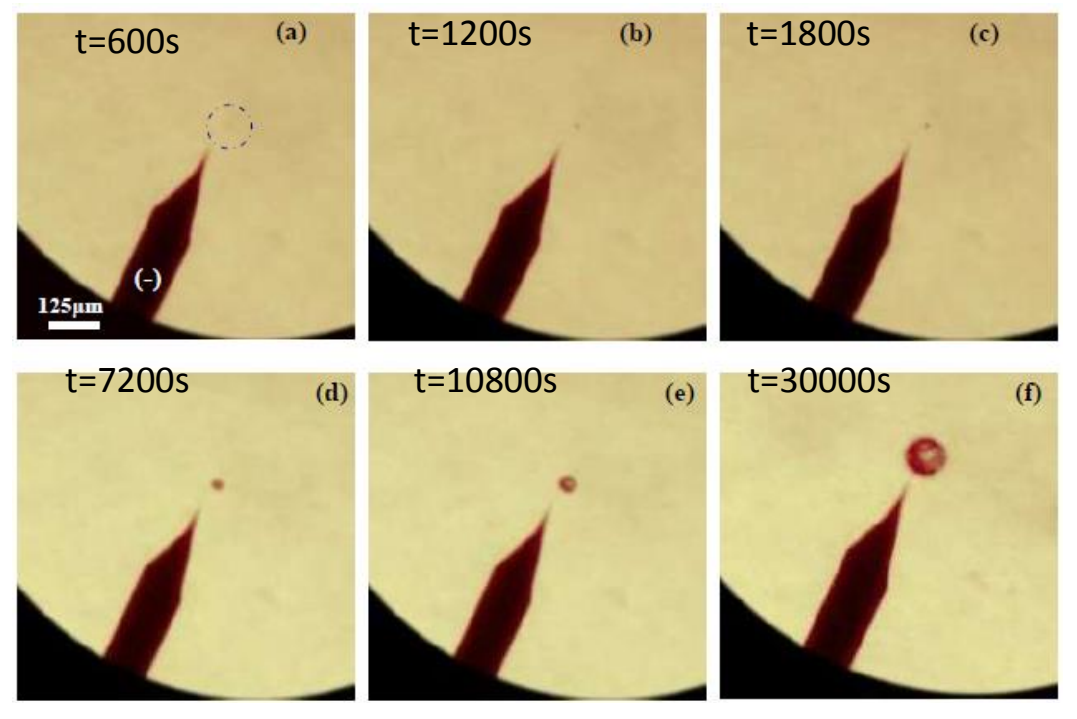

Fig. 14 Time sequence in a supersaturated metastable solution of lysozyme $20 \mathrm{mg} / \mathrm{mL}(\mathrm{NaCl}$ $0.7 \mathrm{M})$. Nucleation is induced by an external localized electric field with a sharp-tip electrode and in agarose gel 1\% gel [16]. Reproduced from Ref [16] with permission from The Royal Society of Chemistry

The optical observation of nucleation gives an induction time shorter than 600s. The nucleated crystal is rough, due to high local supersaturation encountered during nucleation and growth. Growth rate (between 600 and 10800s) is $15 \mu \mathrm{m} / \mathrm{h}$ in agreement with the growth rate obtained by Durbin et al. [63] at high supersaturation for lysozyme. Compared to optical determination of the induction time, the measures of the current during the experiment are clearly more sensitive (Fig. 15) [16]. Indeed the induction time is given by the modification in the slope at 300s.

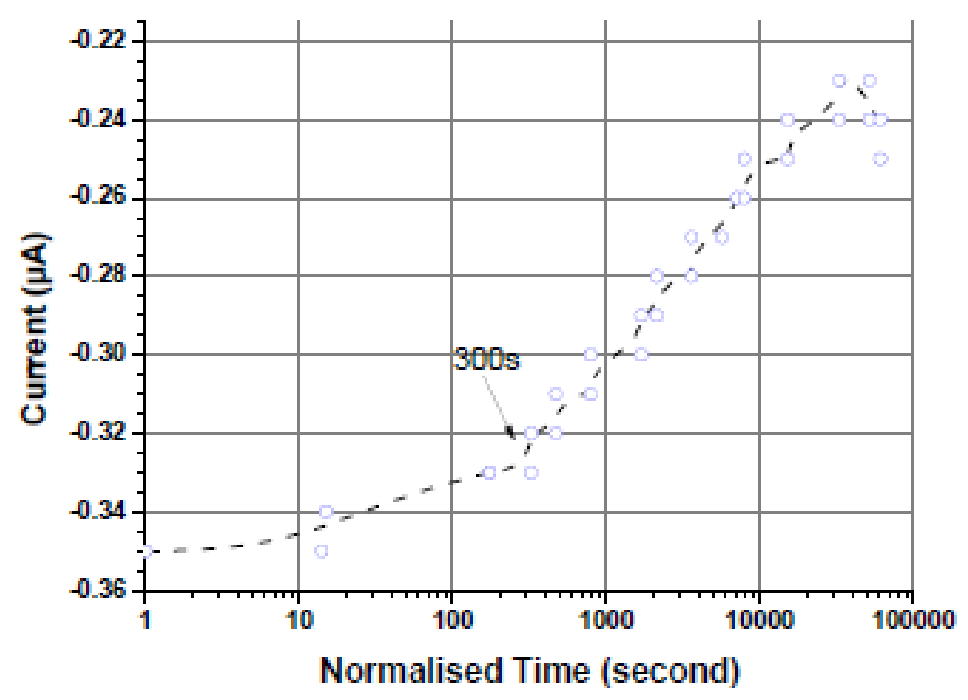

Fig. 15 Evolution of current over time during the experiment, semi-log scale. Line is a guide for the eye. Reproduced from Ref [16] with permission from The Royal Society of Chemistry

To summarize, these techniques of inducing nucleation with fluxes confer a predictive nature on nucleation. Thus the nucleation mechanism can be studied directly on one crystal. 


\section{Conclusion}

Faced with the stochasticity of nucleation, different experimental approaches have been developed to study nucleation. In the first approach, nucleation is allowed to proceed spontaneously and the stochastic nature of results is addressed by statistical studies. Thus the volume is reduced to droplets of $\mathrm{nL}$ to $\mathrm{fL}$ volume that are generated in large numbers. Droplet-based microfluidics and micro-injectors in oil are used. These are compatible with many solvents and involve easy-to-use and material-saving techniques. In the second approach, nucleation is controlled by external electrical or mechanical fields in confined systems. Hence nucleation becomes predictive, triggering nucleation for molecules that are not easy to crystallize, nucleating one crystal per crystallizer and localizing nucleation in space and in time for measurements on the critical nucleus.

All of these methods approach nucleation kinetics through the induction time or the nucleation rate. Moreover, factors affecting the nucleation process (confinement, complexity of solution) are brought to light. These practical approaches show considerable potential to increase understanding and control of the nucleation mechanism.

\section{References}

[1] Kashchiev, D., Nucleation: basic theory with applications, Butterworth-Heinemann, Oxford, 2000.

[2] Candoni, N.; Hammadi, Z.; Grossier, R. ; Ildefonso, M.; Revalor, E.; Ferté, N.; Okutsu, T.; Morin R.; Veesler S. Int. J. Nanotechnol. 2012, Vol. 9, Nos. 3-7.

[3] Hammadi, Z.; Candoni, N.; Grossier, R.; Ildefonso, M.; Morin, R.; Veesler, S. C. R. Phys. 2013, 14, 192-198.

[4] Ildefonso, M. ; Candoni N.; Veesler, S. Crystal Growth \& Design, 2013, 13, 2107-2110.

[5] Ildefonso, M.; Candoni, N.; Veesler, S. Cryst. Growth \& Design, 2011, 11 (5), $1527-1530$.

[6] Ildefonso, M.; Candoni, N.; Veesler, S. Org. Process Res. Dev. 2012, 16, 556-560.

[7] Zhang, S.; Guivier-Curien, C.; Veesler, S.; Candoni, N. Chemical Engineering Science, 2014, CES-D-14-02096 (submitted).

[8] Ildefonso, M.; Revalor, E.; Punniam, P.; Salmon, J. B.; Candoni, N.; Veesler, S. J. Cryst. Growth, 2012, 342 (1), 9-12.

[9] Zhang, S.; Ferté, N.; Candoni, N.; Veesler, S. Org. Process Res. Dev. submitted

[10] Grossier, R.; Hammadi, Z.; Morin, R.; Magnaldo, A.; Veesler, S. Applied Physics Letters, 2011, 98, 091916-091913.

[11] Rodríguez-Ruiz, I.; Hammadi, Z.; Grossier, R.; Gómez-Morales J.; Veesler, S. Langmuir, 2013, 29, 12628-12632.

[12] Grossier, R.; Hammadi, Z.; Morin, R.; Veesler, S. Phys. Rev. Lett. 2011, 107, 025504.

[13] Grossier, R.; Magnaldo, A.; Veesler, S. J. Cryst. Growth, 2010, 312, 487-489.

[14] Hammadi, Z.; Morin, R.; Olives, J. Applied Physics Letters, 2013, 103, 223106.

[15] Hammadi, Z.; Astier, J. P.; Morin R.; Veesler, S. Crystal Growth \& Design, 2007, 7, 1476-1482.

[16] Hammadi, Z.; Grossier, R.; Ikni, A.; Candoni, N.; Morin, R.; Veesler, S. Faraday

Discussions, 2015.

[17] Chernov A.A. Journal of Materials Science: Materials in Electronics, 2001, 12, 437-449.

[18] Gibbs, J. The Collected Works. Vol. 1. Thermodynamics, Yale University Press, 1948.

[19] Dixit, N. M.; Kulkarni A. M.; Zukoski, C. F. Colloids and Surfaces A: Physicochemical and Engineering Aspects, 2001, 190, 47-60.

[20] Selimovic, S.; Jia, Y.; Fraden, S. Crystal Growth \& Design, 2009, 9, 1806-1810. 
[21] Galkin, O.; Vekilov, P.G. J. Phys. Chem. B, 1999, 103, 10965-10971.

[22] Galkin O.; Vekilov, P.G. J. Am. Chem. Soc., 2000, 122, 156-163.

[23] Lekkerkerker, H.N.W. Physica, 1997, A 244, 227-237.

[24] Ten Wolde, P. R.; Frenkel, D. Science, 1997, 277, 1975-1978.

[25] Anderson, V. J.; Lekkerkerker, H. N. W. Nature, 2002, 416, 811-815.

[26] Erdemir, D.; Lee, A.Y.; Myerson, A.S. Chem. Res., 2009, 42(5), 621-629.

[276] Gebauer, D.; Cölfen, H. Nano Today, 2011, 6, 564-584.

[28] Vekilov, P. G. Crystal Growth \& Design, 2004, 4, 671-685.

[29] Erdemir, D.; Lee A. Y.; Myerson, A. S. Accounts of Chem. Res., 2009, 42, 621-629.

[30] Knezic, D.; Zaccaro, J.; Myerson, A. S. J. of Phys. Chem. B, 2004, 108, 10672-10677.

[31] Zettlemoyer, A.C. Nucleation. Marcel Dekker: New York, 1969.

[32] Abraham F.F. Homogeneous nucleation theory. Academic Press: Amsterdam, 1974, 263.

[33] Toschev S. Homogeneous nucleation. Crystal growth: an introduction, Hartman, P., Ed.; North Holland: Amsterdam, 1973, 1-49.

[34] Boistelle, R.; Astier, J.P. J. Cryst. Growth, 1988, 90, 14-30.

[35] Fletcher, N. H. J. Chem. Phys. 1958, 29 (3), 572-576.

[36] Kashchiev, D.; Verdoes, D.; Van Rosmalen, G.M. J. Cryst. Growth, 1991, 110, 373-380.

[37] Tsekova, D.; Dimitrova, S.; Nanev, C.N. J. Cryst. Growth, 1999, 196(2-4), 226-233.

[38] Galkin, O.; Vekilov, P.G. J. Phys. Chem. B, 1999, 103(3), 10965-10971.

[39] Tammann,G. Z. Phys. Chem. 1898, 25, 441-479.

[40] Lee, S.; Wiener, J. Journal of Chemical Education, 2010, 88, 151-157.

[41] Laval, P.; Lisai, N.; Salmon, J-B.; Joanicot, M. Lab Chip, 2007,7, 829-834.

[42] Leng, J.; Salmon, J-B. Lab Chip, 2009, 9, 24-34.

[43] Laval, P.; Salmon, J.-B.; Joanicot, M. J. Cryst. Growth, 2007, 303, 622-628.

[44] Cacioppo, E.; Pusey, M. L. J. Cryst. Growth, 1991, 114, 286-292.

[45] Chayen, N. E. Protein Eng. 1996, 9 (10), 927-929.

[46] Selimovic, S.; Jia, Y.; Fraden, S. Cryst. Growth Des., 2009, 9 (4), 1806-1810.

[47] Ostwald, W. Z. Phys. Chem., 1897, 22, 289.

[48] Ataka, M.; Asai, M. J. Cryst. Growth, 1988, 90, 86-93.

[49] Legrand, L.; Ries-Kautt, M.; Robert, M.-C. Acta Crystallogr. D, 2002, 58, 1564-1567.

[50] Lee, A.Y.; Lee, I.S.; Myerson, A.S. Chem. Eng. Technol. 2006, 29, 281-285.

[51] Mangin, D.; Puel, F.; Veesler, S. Org. Process Res. Dev., 2009, 13, 1241-1253.

[52] Vivares, D.; Veesler, S.; Astier, J.P.; Bonneté F. Cryst. Growth Des., 2006, 6, 287-292.

[53] Duncan, P. B.; Needham, D. Langmuir, 2006, 22, 4190-4197.

[54] Allain, K. ; Bebawee, R.; Lee, S. Cryst. Growth Des., 2009, 9, 3183-3190.

[55] Kashchiev, D. The Journal of Chemical Physics, 2011, 134, 196102-196102.

[56] Grossier R. ; Veesler S. Cryst. Growth Des., 2009, 9, 1917-1922.

[57] Voss, D. Science, 1996, 274, 1325.

[58] Oxtoby, D. W. Nature, 2002, 420, 277-278.

[59] Revalor, E.; Hammadi, Z.; Astier, J. P.; Grossier, R.; Garcia, E.; Hoff, C.; Furuta, K.; Okutsu, T.; Morin, R.; Veesler, S. J. Crystal Growth, 2010, 312, 939-946.

[60] Vivares, D.; Kalera, E. W.; Lenhoff, A. M. Acta Crystallographica Section D, 2005, 61, 819-825.

[61] Chernov, A. A. Phys. Rep. I.M. Lifshitz and Condensed Matter Theory, 1997, 288, 61-75. [62] Gomer, R. Field emission and field ionization, Harvard University press, Cambridge, 1961.

[63] Durbin, S.D.; Feher, G. J. Crystal Growth, 1986, 76, 583-592. 